\title{
Made in America
}

\author{
US $\$ 610$ million has been secured for a US initiative into high-tech manufacturing involving 20 states \\ with the hope of leveraging the capabilities of photonics.
}

Dating back to the industrial revolution, the US has always been a world leader in manufacturing, from weapons, railroads, automobiles and aircraft, to microchips, liquid crystal displays and light-emitting diodes. Although in recent years several companies - including Lenovo,

Caterpillar, GE and Ford - have 'reshored' some jobs back to the US, the overwhelming rise of off-shore manufacturing in other countries, particularly China, may leave some sceptical about the US's future as a powerhouse in manufacturing.

In late July 2015, US Vice President Joe Biden and officials ventured north of Washington to New York to announce a significant investment in manufacturing innovation. US\$110 million in federal Department of Defense (DoD) funding for a photonics-based advanced manufacturing initiative was announced. An additional investment of US\$500 million comes from non-federal sources. In particular, New York Governor Andrew Cuomo has arranged US\$250 million towards the effort.

This is the sixth such initiative in the Nation Network for Manufacturing Innovation. According to the Whitehouse, "each institute is part of a growing network dedicated to securing US leadership in the emerging technologies required to win the next generation of advanced manufacturing" and the initiative "is the kind of investment we need to build on this progress, creating the foundation for American manufacturing leadership for years to come" (http://go.nature.com/kT1rWX). The first initiative in the scheme launched in 2012 and is headquartered in Youngstown, Ohio, and focuses on developing 3D printing technology. Subsequent initiatives target wide-bandgap semiconductors (Raleigh, North Carolina), lightweight technology (Detroit, Michigan), integrated digital design and manufacturing (Chicago, Illinois) and advanced fibre-reinforced polymer composites (Knoxville, Tennessee).

The latest initiative secured more than double the funding of any previous initiative and is centred at the Research Foundation of the State University of New York (RF SUNY), Rochester, New York. "It will bridge advanced research and commercial product development, yielding critical defence and telecommunications

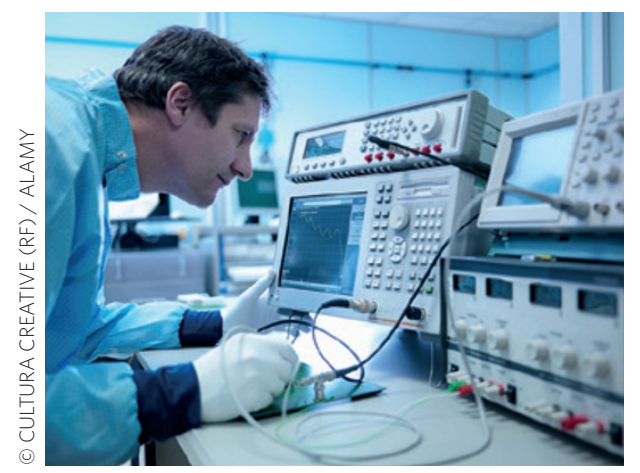

advances, while also investing in education and workforce development to train and position the next generation of manufacturers in integrated photonics," said the Whitehouse.

Funding for the American Institute for Manufacturing Integrated Photonics (AIM Photonics) initiative, headquartered at RF SUNY, is shared among a large and impressive consortium. Fifty-five companies (including Boeing, Corning, GE, Hewlett Packard, Intel, Lockheed Martin, IBM, Raytheon, Seagate, Texas Instruments and others) and twenty universities from the east coast (for example, Boston University, Columbia University, Massachusetts Institute of Technology) to the west coast (for example, Stanford University and several University of California campuses) are included. Not insignificantly, thirtythree community colleges and 16 nonprofit organizations are also incorporated. This photonics initiative is in fact spread across 20 states (New York, California, Massachusetts, Arizona, New Jersey, Florida, Colorado, Maryland, Pennsylvania, Missouri, Indiana, Delaware, Virginia, Minnesota, Idaho, Texas, Washington, Iowa, Connecticut and Oregon.)

The announcements are significant for economic reasons as well as scientific ones. Following the information technology, or dot-com, bubble burst in $2000,40 \%$ of all large factories closed for business over a decade. However, 900,000 manufacturing jobs have been created since February 2010, indicating a job creation rate in manufacturing that is higher than has been seen in decades. According to the Whitehouse, "manufacturing production is up by almost a third since the recession and the number of factories across the US is growing for the first time since the 1990s."

Looking forward, Biden voiced a clear sentiment regarding the need for political co-operation on long-term investment in science and technology for the long-term benefit of the country. "We can make critical bipartisan investments to strengthen manufacturing across the United States, laying a strong foundation for good jobs and economic growth - or we can pull back, letting other countries and their workers take the lead."

US President Barack Obama’s 2016 budget envisages providing the resources to increase to 16 institutes within the year and remarkably there is a ten-year goal of creating up to 45 institutes. Of course none of this is guaranteed, especially in the climate of funding sequestering. The next initiatives in the scheme will focus on "smart manufacturing" and "flexible hybrid electronics".

Rob Clark, senior vice president for research at the University of Rochester and a board member on the AIM Photonics initiative, explained to Nature Photonics that the institute is not necessarily aimed at supporting research on campuses but is focused on enabling critical capabilities in manufacturing desired by the DoD that are enabled by photonics. Clark also explained that approximately US $\$ 130$ million will be managed by the University of Rochester and the Rochester Institute of Technology in collaboration with small and medium enterprises in the area.

"This is a five-year program that was selected because of our ability to sustain this manufacturing operation long after federal funding is gone," Clark told Nature Photonics. "Creating the infrastructure will afford companies opportunities to serve specialized needs of the DoD and find viable commercial sectors for the technology that will enable scale and ultimately reduce cost."

The initiative and financial backing of this level can only be good for photonics and in particular, further development of integrated optics. As Clark points out, integrated photonics may ultimately serve to transform optics in the way that the semiconductor industry transformed electronics decades ago. 